\title{
Valentina Sirangelo
}

\section{Sulla natura lunare di Shub-Niggurath: dalla mythopocin di Howard Phillips Lovecraft a The Moon-Lens di Ramsey Compbell}

ON the LUNAR NatURE OF SHUb-NigGURATH: FROM HOWARD PHILLIPS LOVECRAFT'S MYTHOPOEIA TO RAMSEY CAMPBELL'S THE MOON-LENS

Abstract: The present essay proposes to trace a profile of Shub-Niggurath - one of the terrifying deities conceived by H. P. Lovecraft. The first part illustrates that the double sexuality of Shub-Niggurath - who, though being a Mother Goddess of Fertility, has a more frequent masculine hypostasis, the Goat with a Thousand Young - is informed by the great myth structured upon the distinction between the whole and the part. The second part analyses a Lovecraftian tale by the British writer Ramsey Campbell, The Moon-Lens (1964), which entirely concerns this deity. Beyond the double sexuality of Lovecraftian origin, the Shub-Niggurath of Campbell exhibits several repugnant and hybrid traits which are referable to the lunar law of becoming. Keywords: Mythocriticism, Lovecraft, Campbell, Anglo-American Literature, British Horror Fiction, Moon Archetype, Mother Goddess, Fertility, Initiation, Metamorphosis.

\section{VALENTINA SiRANGELO}

Università della Calabria, Italia valentina.sirangelo@live.com

DOI: $10.24193 /$ cechinox.2018.35.03
"Praise the beast with soul and mind" Morbid Angel, Angel of Disease, 1.17

T a cifra che contraddistingue il gotici$\mathcal{L}_{\text {smo }^{1}}$ di Howard Phillips Lovecraft (1890-1937) deve essere rintracciata nel suo nesso intrinseco con il cosmicismo ${ }^{2}$ che l'autore anglo-americano erige a $\mathrm{Wel}$ tanschauung della propria narrativa orrorifica. "È il cosmicismo", riconosce Barton Levi St. Armand, "a procurare alla narrativa [lovecraftiana] una risonanza unica”, e a permettere a Lovecraft di appropriarsi del "vecchio apparato gotico" 4 e di "investirlo di un nuovo, archetipale significato" Non è un caso che in Supernatural Horror in Literature (L'orrore soprannaturale nella letteratura, 1927) ${ }^{6}$ - lo stesso saggio in cui tratta il gotico come un genre passé rispetto a una ben più suggestiva "tradizione del mistero" [weird tradition] $]^{7}$ - Lovecraft teorizzi in termini bio-antropologici quella "paura cosmica" che attribuisce alla sua opera singolarità e fascino incomparabili:

L'ignoto, che è anche l'imprevedibile, divenne per i nostri primitivi antenati fonte terribile e onnipotente di grazie e calamità elargite all'umanità per 
motivi occulti ed extraterrestri, e quindi appartenenti a sfere di esistenza di cui nulla sappiamo e che non ci riguardano. [...] Gli uomini con menti sensibili a impulsi ereditari tremeranno sempre al pensiero di occulti e insondabili mondi di vita misteriosa che forse vibrano nelle profondità oltre le stelle, o premono spaventosamente sul nostro globo in dimensioni terribili. Con queste premesse, nessuno si stupirà dell'esistenza di una letteratura di paura cosmica ${ }^{8}$.

Stimolando nel lettore un simile "atteggiamento di timoroso ascolto, come a captare $[. .$.$] lo stridere di forme e entità esterne$ sull'estremo del bordo conosciuto dell'universo", il weird tale lovecraftiano si discosta dalla narrativa gotica classica, nonché dalle "storie convenzionali di fantasmi [ghost stories] o di case stregate [haunted-house stories] in cui una singola entità soprannaturale $[. .$.$] infesta un'area spaziale limitata" { }^{10}$. Le mostruosità imperiture di Lovecraft si rivelano "più pericolose e pertanto più terrificanti del vampiro o dello spettro tradizionali"11, poiché esistono al di là del tempo e dello spazio, oltre la portata sensoriale e l'esperienza cosciente dell'uomo ${ }^{12}$ : non sarà mai possibile, pertanto, sapere con esattezza quando una di loro "raggiungerà la nostra dimensione dalla propria per colpire le nostre vite in maniera esecrabile"13. A suggellare l'empio e inscindibile connubio, nell'opera lovecraftiana, di "orrore gotico e terrore cosmico" ${ }^{14}$ è dunque l'irruzione, nella realtà conosciuta, di un pantheon di dèi - e di una pletora di altri esseri deformi - ad essa estranei ed esterni ${ }^{15}$, ma tuttavia in grado di "attraversare i confini dimensionali"16.

Edificato progressivamente e senza alcun intento di organicità ${ }^{17}$ da una fantasia individuale al contempo convulsa e prodigiosa - anziché da una collettività di homines religiosi -, 1'“impianto" [apparatus] $]^{18}$ mitologico di Lovecraft non si sottrae, naturalmente, all'influenza degli archetipi. Se infatti l'archetipo, che figura in formule già elaborate nel mito ${ }^{19}$, è in ultima analisi la "matrice di ogni immaginario"20, esso si manifesterà in formazioni ancora più complesse e criptiche nelle creazioni letterarie della modernità; tanto più in una mythopoeia come quella lovecraftiana, la quale - pur concernendo, nel suo assetto affabulatorio, deità "venerate da culti più antichi dell'uomo"21 - si fonda per definizione sullo stesso semantismo simbolico che ispira il sermo mythicus. Ci proponiamo di illustrare, nel presente saggio, come un archetipo in particolare - quello lunare - si riveli tra le più importanti chiavi di decifrazione per la doppia sessualità e per l'ipostasi caprina di Shub-Niggurath, una delle "divinità infami"22 partorite dall'immaginazione di Lovecraft. Una volta affrontato un simile percorso di ermeneutica "pseudoteologica" 23 , non desterà sorpresa che Ramsey Campbell (1946-) - scrittore britannico appartenente alla "nuova scuola" 24 lovecraftiana - abbia incentrato proprio su Shub-Niggurath un racconto dal titolo The Moon-Lens (La lente lunare, 1964), con il quale restituisce integralmente la multiforme divinità al suo sostrato archetipico.

\section{Il Capro dai Mille Cuccioli e il quarto di luna}

$\mathrm{N}$ onostante la sostanziale mancanza di sistematicità funzionale del pantheon lovecraftiano, a Shub-Niggurath spetta visibilmente il dominio della fertilità, di cui costituisce il "principio cosmico"25: 
ciò comporta la sua collocazione in una delle file più elevate della gerarchia delle entità mitiche create dal visionario di Providence $^{26}$. Nella lettera datata 16 aprile 1936 ad Henry Kuttner, Lovecraft sembra stabilire inequivocabilmente il sesso di Shub-Niggurath, definendola un "demone-femmina", la "moglie" di Yog-Sothoth e la "madre" di Nug e Yeb ${ }^{27}$. Nella narrativa di Lovecraft, la genesi di Shub-Niggurath si situa nelle revisioni [Revision Tales], ossia una categoria di racconti che lo scrittore statunitense, su commissione di terzi, emendava, ampliava o - addirittura - stendeva quasi interamente, sviluppando un abbozzo di trama altrui ${ }^{28}$. Tuttavia, gli studiosi tendono a non includere Shub-Niggurath nel cosiddetto Revision Mythos, che concerne alcuni dèi minori - quali Yig, Nug e Yeb, Ghatanothoa, Rhan-Tegoth, Gnoph-keh, Noth-Yidik e K'thun ${ }^{29}$-, poiché la divinità viene citata più volte anche nei racconti di Lovecraft di maggiore risonanza, assurgendo così ai ranghi del Ciclo Mitico lovecraftiano "canonico" - per intendersi, quello che la critica è incline a denominare Cthulhu Mythos.

Shub-Niggurath appare per la prima volta in The Last Test (L'ultimo esperimento, 1928), revisione di Lovecraft per conto di Adolphe De Castro, in cui è esclusivamente l'oggetto dell'epifonema "Iä! Shub-Niggurath!", proferito immediatamente dopo un riferimento ai "templi sotterranei" sacri a Nug e Yeb, la sua prole ${ }^{30}$. Le stesse urla rituali figurano in due revisioni di Lovecraft per Zealia Bishop, Medusa's Coil (La spira di Medusa, 1939) ${ }^{31}$ e The Mound (Il tumulo, 1940) ${ }^{32}$, nonché in uno dei capolavori appartenenti al suo Ciclo Mitico, The Dunwich Horror (L'orrore di Dunwich, 1930) ${ }^{33}$. In Medusa's Coil e in
The Dunwich Horror, la divinità non viene connotata ulteriormente. In The Mound, invece, si apprende anche che Shub-Niggurath è "la Madre[-di-Tutto] e Moglie di Colui Che Non Deve Essere Nominato"34 e "una specie di sofisticata Astarte" 35 - in linea con la descrizione fornita da Lovecraft della lettera a Kuttner del 1936. Coerente con l'ipostasi femmineo-materna di Shub-Niggurath è anche il titolo "Dea Madre" ${ }^{36}$ di cui è investita nella revisione per Hazel Heald Out of the Aeons (Dagli eoni, 1935). In altre tre revisioni - The Man of Stone (L'uomo di pietra, 1932) $)^{37}$ e The Horror in the Museum (L'orrore nel museo, 1933 ${ }^{38}$, entrambe per la Heald, e The Diary of Alonzo Typer (Il diario di Alonzo Typer, 1938 ${ }^{39}$, per William Lumley -, nonché nel racconto del suo Ciclo Mitico The Dreams in the Witch House (I sogni nella casa stregata, 1933 $)^{40}$, Lovecraft accompagna all'invocazione sacrilega "Iä! Shub-Niggurath!" l'epiteto ${ }^{41}$ "(il) Capro dai Mille Cuccioli" $[(\text { the }) \text { Goat with a Thousand Young }]^{42}$. L'attributo cromatico "Nero" [Black] - a cui, del resto, rimanda etimologicamente "Niggurath" ${ }^{43}$ - viene aggiunto a tale epiteto in due dei racconti più noti del Ciclo Mitico di Lovecraft, The Whisperer in Darkness (Colui che sussurrava nelle tenebre, 1931) ${ }^{44}$ e The Thing on the Doorstep (La cosa sulla soglia, 1937) ${ }^{45}$. In The Whisperer in Darkness, ancora, l'epiteto di Shub-Niggurath si arricchisce della specificazione "dei Boschi" [of the Woods] ${ }^{46}$; sempre in The Whisperer in Darkness, si attesta un secondo epiteto della divinità: "Signore dei Boschi" [Lord of the Woods] $]^{47}$ - unica occorrenza nell'intero corpus lovecraftiano.

Dinanzi alle apposizioni "Signore" e "Capro" - la prima d'eccezione, la seconda molto frequente - insorgono, non senza 
ragione, gravi perplessità circa l'univocità del sesso di Shub-Niggurath ${ }^{48}$. Si nota, difatti, nella letteratura critica su Lovecraft, una continua oscillazione nell'uso del pronome personale per la deità in questione. Lin Carter la presenta al femminile quando, nella sua trattazione di The Dunwich Horror, osserva che "una nuova divinità, Shub-Niggurath, fa il suo [her] esordio nella stampa in questo racconto" ${ }^{49}$. Philip A. Shreffler, pur classificandolo in principio come un "dio" ${ }^{50}$, più specificamente un "dio della fertilità" [fertility god $]^{51}$ - formula scelta in seguito anche da Gianfranco de Turris e Sebastiano Fusco nella critica italiana ${ }^{52}$ - prosegue più cautamente: "Lui (o lei) è da collocarsi in alto nel pantheon"53. Robert M. Price vi attribuisce perlopiù il pronome "lei" $[s h e]^{54}$ definendola, al contrario di Shreffler, "dea della fertilità" [fertility goddess] $]^{55}-$, ma quando sottolinea la necessità di far luce sulla sua identità al di là del suo nome, alterna al femminile addirittura il neutro: "Chi o cosa è Shub-Niggurath e cosa lei [she] o esso [it] faceva?" 56 .

L'ipostasi maschile di Shub-Niggurath rappresenta, in particolare, uno dei più assillanti enigmi irrisolti della pseudoteologia lovecraftiana: se da una parte Shub-Niggurath è un "demone-femmina", la "Dea Madre", la "Madre-diTutto", dall'altra è il "(Nero) Capro (dei Boschi) dai Mille Cuccioli" e il "Signore dei Boschi”, identità che implica rispetto alla "Dea" uno slittamento di sesso. Nel tracciare lo status quaestionis, procederemo cronologicamente a ritroso. Giuseppe Lippi, nella sua traduzione dell'epistolario di Lovecraft, tentava di aggirare l'ostacolo rendendo goat con "capra" 57 , poiché - spiega - "a rigor di logica, visto che si allude alla prole della bestia, sarebbe stato meglio immaginarla femmina fin dal primo momento"58; una simile scappatoia, tuttavia, non appare affatto convincente, anche perché confutata dall'epiteto secondario - e certamente maschile - "Signore dei Boschi" in The Whisperer in Darkness. Rodolfo A. Ferraresi proponeva, in "The Question of Shub-Niggurath" - un saggio del 1985 - la teoria secondo cui il Capro non sia Shub-Niggurath, ma rappresenti il mero symbolon, in ambito rituale, della fertilità che le compete. A supporto della sua tesi, Ferraresi evidenziava come, in un passo di Out of the Aeons, il personaggio di T'yog sia presentato come "alto sacerdote di Shub-Niggurath e guardiano del tempio [di rame] del Capro dai Mille Cuccioli"59. $\mathrm{Si}$ tratta dell'unico caso, nella narrativa di Lovecraft, in cui Shub-Niggurath e il Capro non vengono giustapposti, bensì disgiunti ${ }^{60}$ :

Sembra che $\mathrm{H}$ [oward] $\mathrm{P}$ [hillips] $\mathrm{L}$ [ovecraft] tratti due entità separate: Shub-Niggurath [...] è venerata attraverso i riti di un capro - che, a sua volta, ha il proprio tempio di rame. [...] L'evidenza più convincente, comunque, che il Capro Nero sia un'entità distinta da Shub-Niggurath deve essere ricercata nel fatto che il "Capro Nero" è designato come maschile: ciò perché, nell'inno agli Antichi in The Whisperer in Darkness, viene chiamato «Signore dei Boschi» ${ }^{61}$.

Secondo la posizione di Ferraresi, pertanto, "(il) Capro dai Mille Cuccioli" non è un epiteto di Shub-Niggurath, ma un'esclamazione separata; d'altronde - ritiene lo studioso - i sessi opposti della Dea e del 
Capro non possono che corroborare l'ipotesi. Appena un anno prima, nel 1984, all'interno di un saggio su Nug e Yeb, anche Will Murray sollevava il problema della doppia sessualità di Shub-Niggurath, proponendo una - forse meno netta - separazione ontologica tra la Dea Madre femminile da un lato e il Capro Nero maschile dall'altro:

E possibile che l'immagine del capro sia [solo] rappresentativa delle proprietà di Shub-Niggurath come dea della fertilità ("la Madre-diTutto", come la chiama Lovecraft in The Mound) e non vada presa alla lettera $^{62}$.

La Dea e il Capro si rivelano, invece, tutt'altro che ontologicamente incompatibili, se contemplate attraverso il prisma dell'archetipologia lunare. Identificando Shub-Niggurath con la luna, sarà possibile motivare il suo sesso duplice, superando così la dicotomia mutuamente esclusiva delle ipostasi - entrambe degne della deità tutrice della fertilità universale - della Madre di Ogni Cosa e del Capro dalla Prole Innumerevole.

La luna è "il simbolo [...] privilegiato della sfera matriarcale" 63 . Molto spesso, la Dea Madre - e non solo la cananeo-fenicia Astarte ${ }^{64}$, alla quale Shub-Niggurath è assimilata da Lovecraft in The Mound - è la luna ${ }^{65}$, poiché "questo simbolo concerne la divinità della donna e la potenza fecondante della vita, incarnate nelle divinità della fecondità vegetale e animale, fuse nel culto della Grande Madre (Magna Mater)"66. Classificata come la Dea Madre assoluta nel pantheon di Lovecraft, Shub-Niggurath può dunque riconoscersi come la sua unica deità conformata sul modello archetipico della luna, "fonte di ogni fertilità" ${ }^{67}$.

"Un attributo lunare" - scrive Mircea Eliade - "rimane sempre trasparente: il prestigio della fecondità, della creazione periodica, della vita inesauribile" ${ }^{68}$. La prolificità illimitata, prerogativa dell'archetipo Luna, si rintraccia soprattutto nelle qualità possedute dall'ipostasi maschile di Shub-Niggurath - il Capro detto "dai Mille Cuccioli" - che è quella problematica, in quanto entra in contraddizione con la sua ipostasi femmineo-materna. Tuttavia, si osservi anzitutto che, in virtù della diffusa "coesistenza dei simboli lunari con quelli della fecondità" ${ }^{9}$, le corna - di cui il Capro è provvisto - rientrano nella costellazione simbolica della Dea Madre:

La fecondità degli animali, come la fertilità delle piante, è soggetta alla Luna. [...] Le corna [...], che caratterizzano le grandi divinità della fecondità, sono un emblema della Magna Mater divina. Dovunque compaiono, [...] segnano la presenza della Grande Dea della fecondità ${ }^{70}$.

Uno dei titoli di Astarte è proprio Ashtaroth Qarnajim, "Astarte dalle due Corna"71 - attributo che la dea possiede in una documentata iconografia ${ }^{72}$. Allo stesso modo, quella "sofisticata Astarte" che è Shub-Niggurath si manifesta spesso, a riprova della sua smisurata fecondità, sotto forma di una bestia dotata di un paio di corna: il Capro.

Rimane, tuttavia, il cavillo riguardante la maschilità del "Capro dai Mille Cuccioli". Se il Capro rivela epifanicamente la presenza di Shub-Niggurath, deità della fertilità sconfinata, senza tuttavia essere 
altro da lei, perché mai, allora, è maschile? Perché il rapporto dialettico tra la Dea e il Capro è quello tematizzato dal grande mito strutturato sulla "distinzione tra la «totalità» [whole], personificata come la Grande Dea Madre, e «la parte», personificata come il suo figlio-amante"73, vale a dire tra la luna piena e il quarto di luna:

La diversità degli aspetti attribuiti alla luna, a volte come maschile rapportato al femminile, a volte come femminile rapportato al maschile [...] si esprime anche nella diversità delle sue fasi $(\mathrm{ad}$ esempio come falce crescente o calante può essere considerata maschile e come luna piena femminile) $)^{74}$.

Pertanto, se Shub-Niggurath quale "Dea Madre" è la luna piena, totale e femminile, Shub-Niggurath quale "Capro dai Mille Cuccioli" sarà il quarto di luna, parziale e maschile, di cui la Dea è potenzialmente gravida - ragione per la quale non è altro da lei. Sebbene ciò non emerga mai dalla fenomenologia del divino lovecraftiana, il modello mitico-archetipico del Capro è quello del Dio-Figlio-e-Paredro della Grande Dea ${ }^{75}$ :

L'iconografia sottolinea sempre l'ambivalenza delle divinità assimilabili alla luna [...]. Una variante $\mathrm{d}[\mathrm{el}]$ doppio uso mitico della divinità è rappresentata nell'iconografia dal mito dell'androgino. [...] L'epifania maschile [della divinità androgina] è quella del Figlio femminoide. La maggior parte delle divinità lunari $[\ldots]$ esibisce una doppia sessualità $[. .$.$] . Nascono così [. .$. le curiose dee barbute, quali la Cibele frigia, la Didone-Astarte cartaginese, la Fortuna e la Venus barbata romana ${ }^{76}$.
Se la religione comparata contemplasse anche la mythopoeia lovecraftiana, questa schiera di divinità dai tratti sessuali ambigui per via del loro sostrato lunare potrebbe pertinentemente includere anche Shub-Niggurath, Dea Madre la cui più frequente ipostasi è quella maschile del Capro - a cui ci si appella, occasionalmente, come a un "Signore"77. D'altro canto, Ferraresi stesso notava che "Astarte, a cui $\mathrm{H}$ [oward] $\mathrm{P}$ [hillips] L[ovecraft] collega Shub-Niggurath, veniva talvolta venerata sotto la forma maschile di Athar"78, pur non cogliendo che tale doppiezza si deve al sostrato lunare della divinità cananeo-fenicia stessa ${ }^{79}$.

Ferraresi sosteneva che la fonte dello slittamento sessuale di "Shub-Niggurath che passa dal maschile al femminile" 80 - che, si ricordi, concepisce come due entità ontologicamente distinte - debba cercarsi nel Pan di Arthur Machen: nello sconcertante racconto The Great God Pan (Il Grande Dio Pan, 1894) dello scrittore gallese - principale ispiratore di Lovecraft $^{81}$-, la macabra creatura è difatti in grado di compiere trasformazioni infinite, non ultima quella "da uomo a donna" 82 . I1 prolifico "Capro dai Mille Cuccioli" è in effetti riconducibile - via Machen o meno - al dio greco minore Pan, anche lui "cornuto" ${ }^{83}$, quintessenza della "sensualità primaria irreprimibile" ${ }^{84}$ e signore del bosco ${ }^{85}$. Tuttavia, la correlazione di Shub-Niggurath al solo Pan sulla base di uno studio delle fonti letterarie di Lovecraft o alla luce del suo interesse per il pantheon greco e non su una più ampia prospettiva comparativo-mitologica e archetipologica - è limitativa. A un raffronto bilaterale tra Shub-Niggurath e Pan deve piuttosto sostituirsi una riconsiderazione di entrambi 
nella prospettiva simbologica del corno che li caratterizza, isomorfo al quarto di luna, a sua volta isomorfo al fallo - simbolo del principio Maschile: come sottolinea Gilbert Durand, "è il corno, imputrescibile e dalla forma oblunga esplicitamente suggestiva, a simboleggiare in maniera eccellente la potenza virile" ${ }^{\text {. }}$. Shub-Niggurath quale "Capro dai Mille Cuccioli" non è dunque tanto un novello Pan, ma piuttosto rientra - così come vi rientra Pan - nella categoria, propria della mitologia comparata, del dio cornuto-fallico promotore della fertilità ${ }^{87}$ non di rado dotato di "prestigi lunari" 88 in virtù dell'isomorfismo triplice fra il quarto lunare, il membro virile e il corno caprino.

\section{Le iniziazioni di Goatswood: la luna multiforme}

Shub-Niggurath godette - e gode - di Suna fortuna notevole sia tra i seguaci che tra i continuatori della mythopoeia di Lovecraft ${ }^{89}$. Tra questi ultimi, il britannico Ramsey Campbell - il quale, in realtà, è "influenzato dal goticismo di Lovecraft" soltanto nella sua narrativa giovanile consacra completamente a Shub-Niggurath il racconto The Moon-Lens (La lente lunare, 1964) che, con la sua stessa impostazione simbolico-tematica, supporta e comprova la nostra tesi circa la rilevanza dell'archetipo Luna nella conformazione di tale divinità.

The Moon-Lens chiude la prima raccolta di racconti di Campbell, The Inhabitant of the Lake and Less Welcome Tenants (L'abitatore del lago e inquilini meno benaccetti, 1964) ${ }^{91}$; è ritenuta da Campbell stesso la sua ultima storia lovecraftiana ${ }^{92}$, giacché a partire dalla raccolta successiva, Demons by Daylight (Demoni all'alba,
1973), lo scrittore "iniziò a sviluppare un universo fittizio più personale" ${ }^{\text {. }}$. Il setting di The Moon-Lens è Goatswood, cittadina di invenzione collocata da Campbell nella Severn Valley, in Inghilterra ${ }^{94}$ : i componenti nominali di cui consta tale toponimo immaginario, "capro" [goat] e "bosco" [wood], richiamano alla memoria, rispettivamente, l'ipostasi maschile di Shub-Niggurath e i "Boschi" di cui la divinità in questione è il "Signore".

Lappassionato di libri antiquari Roy Leakey rimane bloccato, una sera, nella sperduta e sinistra Goatswood, presso la cui stazione è dovuto necessariamente scendere per effettuare l'unico cambio di treno previsto nel suo itinerario da Brichester ad Exham. Costretto a pernottarvi, Leakey finisce col diventare vittima di un sacrificio umano per l'abominevole deità venerata dagli abitanti del luogo, Shub-Niggurath - risvolto che esaspera il motivo lovecraftiano dell'"eroe [che] partecipa suo malgrado alla celebrazione di un culto immondo" 95 . Lo Shub-Niggurath campbelliano viene evocato attraverso un bizzarro marchingegno - la lente lunare - atto a proiettare la luce selenica sulla spoglia collina in cui è imprigionato, per spalancarla e lasciargli via libera ${ }^{96}$. La nostra esegesi del seguito dell'iniquo rito di Goatswood si articolerà in due tappe cardinali: la morte iniziatica di Leakey sotto forma di discesa negli inferi, analoga a quella della luna durante la fase del novilunio ${ }^{97}$; e la rinascita iniziatica di Leakey come metamorfosi a carattere ibrido, che ripete in forma degradata la fase della luna crescente.

Prima della sua evocazione, Shub-Niggurath viene designato, in The Moon-Lens, al maschile, come il "Capro". L'albergatore 
di Goatswood, dopo aver rinchiuso Leakey a chiave in camera, elenca - in uno sproloquio minaccioso e delirante - una sequela di divinità e rituali che, nella storia universale delle religioni e in un'area geografica che si estende dall'Egitto al Centro America, alluderebbero a suo dire all'adorazione dell'ipostasi caprina di Shub-Niggurath sotto nomi diversi ${ }^{98}$ :

"Sai da dove prende il proprio nome Goatswood?" disse una voce dietro di lui.

Leakey si girò. Non c'era nessuno nella stanza con lui.

"Hai mai sentito parlare del Caprone di Mendes?” proseguì la voce lentamente - capì - da dietro la porta. "Sai che cosa appariva ai sabba delle streghe? Conosci la Terra del Caprone nei Pirenei, o il Grande Dio Pan? E che mi dici del Dio Proteo? E del Capro Nero dei Boschi dai Mille Cuccioli?" [...]

"Il capro è stato qui in tutte le ere, sai" continuò la voce. "Il capro nero che appariva nel circolo delle sette in Spagna - il Prato del Capro dove si incontravano i maghi baschi - e il diavolo appare sempre come un animale ibrido. Perché pensi che i preti di Giove offrivano un capro bianco alle Idi? - ma non saprai dei complementi cosmici... E non hai idea della base del rituale della donna-capro ad Haiti, o di quale orrore si celi dietro il mito del Vello D’Oro..." 99 .

Tuttavia, quando Shub-Niggurath si accinge ad apparire al culmine della cerimonia della lente lunare, l'esclamazione del sacerdote adibito all'empio rito - nel quale
Leakey riconosce proprio il folle albergatore - adopera anche il pronome personale "lei":

"Lui sta arrivando! Lei sta arrivando!" gridò con quella voce lenta e pesante. "Liberate la strada!".

Così, con orrore di Leakey, la folla iniziò a cantare: "Astarte - Ashtaroth - Magna Mater... Iä! Shub-Niggurath! Gorgo, Mormo, luna dai mille volti, guarda con favore ai nostri sacrifici... Ariete dalle Mille Pecore, riempici del tuo seme affinché in più possano venerarti...Gof'nn hupadgh Shub-Niggurath...".

Il disco di luce lunare concentrata avanzava lentamente su per la collina, mentre il sacerdote in abito rituale manipolava le corde. All'improvviso titubò e si fermò, emise un grido inarticolato, e la folla si azzittì. In quel silenzio Leakey sentì un indistinto trambusto irrequieto, come di qualcosa distante - e vasto.

Allora la collina si spalancò ${ }^{100}$.

La lode innalzata dagli adepti di Shub-Niggurath si allinea con l'enfasi della sua doppia sessualità: pur dichiarando la sua identità con più divinità femminili lungo le ere storico-religiose (prima tra tutte, la Astarte di lovecraftiana memoria), è all'ipostasi maschile che si rivolgono - 1"Ariete dalle Mille Pecore" [Ram with a Thousand Ewes] ${ }^{101}$, variante del "Capro dai Mille Cuccioli” di Lovecraft - pregando di essere fecondati affinché possano moltiplicarsi.

La porta sulla collina, una volta aperta, permette di penetrare la Terra, la quale come la luna piena o totale - incarna la 
Magna Mater $^{102}$. Lo sventurato Leakey viene ghermito da Shub-Niggurath quale abominevole "figlio" lunare evocato e partorito dagli abissi uterini della Grande Madre selenico-tellurica - e da lui forzato a compiere una discesa agli inferi che riproduce ritualmente quella della luna durante le tre notti di tenebre:

Per tre notti il cielo resta buio; ma, appunto come la Luna rinasce la quarta sera, anche i morti acquisiranno una nuova modalità di esistenza. La morte $[. .$.$] non è un'estinzione, è$ modificazione - di solito provvisoria del piano vitale. La morte partecipa a un altro genere di "vita". E, in seguito al fatto che questa "vita della morte" è convalidata e valorizzata dalla "storia" della Luna e (data la corrispondenza Terra-Luna, popolarizzata dalla scoperta dell'agricoltura) da quella della Terra, i defunti passano dalla Luna o tornano sottoterra per rigenerarsi e assimilare le forze necessarie a una nuova esistenza ${ }^{103}$.

Mentre lo Shub-Niggurath di Lovecraft non appare mai come una "presenza fisica" 104 ed è "scarsamente definito" 105 , lo Shub-Niggurath di Campbell si manifesta materialmente in The Moon-Lens ed è descritto nel dettaglio. Tuttavia, lo stesso albergatore annuncia a Leakey che l'abominio "non è fatto come un capro"106 come invece ci si aspetterebbe, in quanto "figlio" e parte dalla Dea Madre selenico-tellurica. Una volta uscito dalla collina, conferma i tremendi presentimenti di Leakey: è la rivoltante "cosa"107 ibrida che aveva osservato poco prima in una fotografia nella sua stanza d'albergo ${ }^{108}$, ossia un "pilastro di pelle bianca sorretto da zampe ossute dotate di molte articolazioni che terminavano con grandi rigonfiamenti circolari"109, il quale "non aveva braccia, ma soltanto tre spine dorsali che sprofondavano nel terreno"110 e un'orrenda "testa - formata da spire spesse di gelatina bianca ricoperte da grigi occhi acquosi, con al centro un enorme becco dentato"111. Un simile aspetto all'insegna della multiformità più ripugnante - malgrado la "molteplicità brulicante"112 caratterizzi anche il tipico mostro lovecraftiano - deve essere codificato secondo la "norma lunare [del] divenire" 113 e la "polivalenza delle raffigurazioni della luna" ${ }^{114}$. Tali prerogative erano state anticipate implicitamente, in primo luogo, nel delirio verbale dell'albergatore, il quale menziona, tra le ipostasi di Shub-Niggurath nel corso dei secoli, anche il dio greco Proteo, in grado di assumere qualsiasi forma ${ }^{115}$; $\mathrm{e}$ in secondo luogo, dalla lode a Shub-Niggurath da parte dei suoi adoratori, i quali citano unemblematica "luna dai mille volti"116.

La multiformità lunare si rintraccia anche negli esiti dell'iniziazione già sperimentata dagli abitanti di Goatswood, i quali sin dal principio avevano generato in Leakey l'impressione di celare, sotto le proprie vesti esageratamente voluminose, "le più rivoltanti deformita" "117. Evidentemente, è dopo aver completato la catabasi collinare e dunque l'iniziazione ai "misteri" lunari di Shub-Niggurath che gli adepti i quali si configurano, in The Moon-Lens, come i suoi proverbiali "cuccioli" - sono rinati orrendamente mutati:

Noi siamo andati laggiù, nel suo luogo, in una regione che non ti descriverò, e per vivere più a lungo dobbiamo... 
cambiare. Ne avrai sentito comunque parlare in un altro modo - i cuccioli del Capro Nero? gof'nn hupadgh Shub-Niggurath? ${ }^{118}$.

Quando Leakey raggiunge il punto più basso del mondo sotterraneo - ossia, il Centro del cammino iniziatico quale discesa agli inferi, dopo il quale comincia la risalita ${ }^{119}$ - il sacerdote gli annuncia: "È qui che veniamo per conquistare l'immortalità [...] e adesso diventerai come noi..." ${ }^{120}$, ossia, immortale come la luna, ma trasfigurato:

La Luna non conosce morte. [...] È quindi facile capire la parte rappresentata dalla Luna nelle cerimonie di iniziazione, che consistono precisamente nello sperimentare una morte rituale seguita da "rinascita" e con le quali l'iniziato reintegra la sua vera personalità di "uomo nuovo"121.

Mentre di norma 1"“uomo nuovo" post-iniziazione accede a "un modo superiore di esistenza"122, 1'"uomo nuovo" iniziato ai misteri di Shub-Niggurath, pur ottenendo l'immortalità lunare, precipita nella degenerazione dell'ibridismo. La mano del "nuovo" Leakey, la cui vista sigilla The Moon-Lens, è "di un nero sfavillante [...] ricoperta di linee - simile a quella di un artiglio di uccello fatto di legno", e "non sembr[a] affatto una mano umana"123; su di lui si apprende inoltre - ben prima di conoscerne la causa - che emana un "odore di rettile"124. L'ibridismo contraddistingue già i mostri di Lovecraft ${ }^{125}$, ma nella costellazione simbolica tracciata da Campbell in The Moon-Lens esso acquisisce una solida base archetipica, dettata dalla multiformità e dalla metamorfosi lunare. Rimane, ad ogni modo, un ibridismo "blasfemo" in senso lovecraftiano, in quanto "trasgressione $\mathrm{o}$ disfacimento delle categorie accettate della realtà"126, ossia la violazione dei confini tra uomo e animale - peraltro suscettibile di simboleggiare il divenire lunare:

Lo schema ciclico eufemizza l'animalità [...]. Il "mostro" è in effetti simbolo di totalizzazione, di inventario completo delle possibilità naturali; da questo punto di vista ogni animale lunare, anche il più umile, è un assemblaggio mostruoso. Si può dire che tutto il meraviglioso teratologico è un meraviglioso totalizzante e che questa totalità simboleggia sempre la potenza fasta e nefasta del divenire ${ }^{127}$.

Sul finale di The-Moon Lens, il dottor James Linwood - al quale il disperato Leakey ha narrato la sua rovinosa avventura - impazzisce alla vista delle sembianze dell'uomo, dopo averlo invitato a spogliarsi per farsi visitare e per dimostrare di non aver mentito. Farneticando, afferma che il suo paziente "era «orribilmente mutato», e sembrava connesso al «Grande Dio Pan», «una rinascita nella vagina di Shub-Niggurath», «una fluttuazione della forma»"128 - riferimenti in cui Campbell (dopo un celere omaggio al Pan macheniano) riassume la morte e la rinascita iniziatica dall'utero della Dea Madre selenico-tellurica e l'esito degradato di un'iniziazione modellata sul divenire lunare.

\section{d}

Mentre Lovecraft non correla mai esplicitamente Shub-Niggurath alla luna, Campbell riproduce più fedelmente la 
costellazione simbolica in cui si inserisce, sviluppando il suo Shub-Niggurath su un piano di caratterizzazione più avanzato: nella fattispecie, lo scrittore britannico estende la doppiezza sessuale della divinità
- che semplicemente trapelava dagli indizi del suo maestro, e che è motivata dall'archetipologia lunare - a una molteplicità che è conseguenza diretta della "modalità lunare per eccellenza, quella del mutamento" 129 .

\section{Bibliografia}

Anne Baring, Jules Cashford, The Myth of the Goddess. Evolution of an Image, London, Penguin, 1993. Germain Bazin, "Formes démoniaques", in Satan, Coll. "Études Carmelitaines", n. 6, Paris, Desclée de Brouwer, 1948, p. 507-520.

Clive Bloom, "Horror Fiction: In Search of a Definition", in David Punter (ed.), A New Companion to the Gothic, Malden (MA), Wiley-Blackwell, 2012, p. 211-223.

Pierre Brunel, Juliette Vion-Dury (dir.), Dictionnaire des mythes du fantastique, Limoges, Pulim, 2003. Gavin Callaghan, H. P. Lovecraft's Dark Arcadia: The Satire, Symbology and Contradiction, Jefferson (NC) and London, McFarland \& Company, 2013.

J. Ramsey Campbell, The Inhabitant of the Lake and Less Welcome Tenants, Sauk City, Arkham House, 1964.

Ramsey Campbell, The Moon-Lens, in Robert M. Price (sel. and ed.), The Shub-Niggurath Cycle: Tales of the Black Goat with a Thousand Young, Oakland (CA), Chaosium, 1994, p. 41-53.

Ramsey Campbell, Stefan R. Dziemianowicz, Sunand T. Joshi, The Core of Ramsey Campbell: A Bibliography and Reader's Guide, West Warwick (RI), Necronomicon Press, 1995.

Ramsey Campbell, "A Word from the Author", in Id., The Inhabitant of the Lake and Other Unwelcome Tenants, Hornsea, PS Publishing, 2011, p. VII-IX.

Ramsey Campbell, The Inhabitant of the Lake and Other Unwelcome Tenants, Hornsea, PS Publishing, 2011.

Lin Carter, Lovecraft: A Look Behind the "Cthulhu Mythos», New York, Ballantine Books, 1972.

Jean Chevalier, Alain Gheerbrant, Dizionario dei simboli, Milano, BUR, 2011, 2 voll.

Izak Cornelius, The Many Faces of the Goddess: The Iconography of the Syro-Palestinian Goddesses Anat, Astarte, Qedeshet, and Asherah c. 1500-1000 BCE, Fribourg, Academic Press, and Göttingen, Vandenhoeck \& Ruprecht, 2004.

Gary William Crawford, "Urban Gothic: The Fiction of Ramsey Campbell", in Darrell Schweitzer (ed.), Discovering Modern Horror Fiction, Berkeley Heights (NJ), Wildside Press, 1999, vol. I, p. 13-20.

Gary William Crawford (ed.), Ramsey Campbell: Critical Essays on the Modern Master of Horror, Lanham (MD), Scarecrow Press, 2014.

August Derleth, "Genesi e struttura dei «Miti di Cthulhu»", in Id. (a cura di), I Miti di Cthulhu, introduzione, prologo, parte prima, epilogo, bibliografia, note e commenti di Gianfranco de Turris, Sebastiano Fusco, Roma, Fanucci, 1975, p. 15-21.

August Derleth, "Le «revisioni» di Lovecraft", in Howard Phillips Lovecraft, Nelle spire di Medusa, traduzione integrale di Roberta Rambelli, Roma, Fanucci, 1976, p. 17-19.

Gianfranco de Turris, Sebastiano Fusco, Howard Phillips Lovecraft, Firenze, La Nuova Italia, 1979.

Gilbert Durand, "Méthode archétypologique: de la mythocritique à la mythanalyse", in Id., Champs de l'imaginaire, textes réunis par Danièle Chauvin, Grenoble, Ellug, Université Stendhal, 1996, p. 133-156. Gilbert Durand, Le strutture antropologiche dell'immaginario. Introduzione all'archetipologia generale, Bari, Edizioni Dedalo, 2009.

Stefan Dziemianowicz, "Mastering Darkness: An Interview with Ramsey Campbell”, in Gary William Crawford (ed.), Ramsey Campbell: Critical Essays on the Modern Master of Horror, Lanham (MD), Scarecrow Press, 2014, p. 113-146. 
Mircea Eliade, Mito e realtà, Torino, Borla, 1966.

Mircea Eliade, Trattato di storia delle religioni, Torino, Bollati Boringhieri, 2008.

Rodolfo A. Ferraresi, “The Question of Shub-Niggurath”, in Crypt of Cthulhu, n. 35, 1985, p. 17-18, p. 22.

James G. Frazer, Il ramo d'oro. Studio sulla magia e sulla religione, Roma, Newton Compton, 2006.

Meade Frierson III, Penny Frierson (eds.), HPL: A Tribute to Howard Phillips Lovecraft, Birmingham (AL), The Editors, 1972.

Renzo Giorgetti, Archetipi lovecraftiani: l'Eterno Femminile, Stienta, Edizioni Diversa Sintonia, 2012.

Armando Gnisci, "Howard Phillips Lovecraft", in Elémire Zolla (a cura di), I contemporanei: Novecento americano, Roma, Lucarini, 1986, vol. I, p. 553-562.

Giulio Guidorizzi, Il mito greco, Milano, Mondadori, 2010, 2 voll.

Daniel Harms, "Great Old Ones", in Id., Encyclopedia Cthulhiana, Oakland (CA), Chaosium, 1994, p. 86-88.

Daniel Harms, "Outer Gods", in Id., Encyclopedia Cthulhiana, Oakland (CA), Chaosium, 1994, p. 161-162.

Daniel Harms, "Ram with a Thousand Ewes", in Id., Encyclopedia Ctbulbiana, Oakland (CA), Chaosium, 1994, p. 175.

Daniel Harms, "Shub-Niggurath", in Id., Encyclopedia Cthulhiana, Oakland (CA), Chaosium, 1994, p. 192-193.

William Hughes, David Punter, Andrew Smith (eds.), The Encyclopedia of the Gothic, Malden (MA), Wiley, 2016.

Chris Jarocha-Ernst, A Cthulhu Mythos Bibliography and Concordance, Seattle (WA), Armitage House, 1999.

Sunand T. Joshi (ed.), H. P. Lovecraft: Four Decades of Criticism, Athens (OH), Ohio University Press, 1980.

Sunand T. Joshi, David E. Schultz (eds.), An Epicure in the Terrible: A Centennial Anthology of Essays in Honor of H. P. Lovecraft, Cranbury (NJ), Associated University Presses, 1991.

Sunand T. Joshi, A Dreamer and a Visionary: H. P. Lovecraft in His Time, Liverpool, Liverpool University Press, 2001.

Sunand T. Joshi, Ramsey Campbell and Modern Horror Fiction, Liverpool, Liverpool University Press, 2001.

Sunand T. Joshi, "Master and Pupil: August Derleth and Ramsey Campbell's First Book", in Gary William Crawford (ed.), Ramsey Campbell: Critical Essays on the Modern Master of Horror, Lanham (MD), Scarecrow Press, 2014, p. 101-112.

Sunand T. Joshi, David E. Schultz, "Cthulhu Mythos", in Id., An H. P. Lovecraft Encyclopedia, Westport (CT), Greenwood Press, 2001, p. 50-55.

Carl Gustav Jung, Gli archetipi dell'inconscio collettivo, Torino, Bollati Boringhieri, 2011.

Alexander Haggerty Krappe, La genèse des mythes, Paris, Payot, 1938.

Francis Lacassin, "Lovecraft et les trous de la toile peinte", in Cabiers de l'Herne, n. 12, 1969, p. 106-110.

Franco La Polla, "Lovecraft, il gotico e i segni cosmici", in Il Verri, n. 37, 1971, p. 68-75.

Fritz Leiber Jr., "A Literary Copernicus", in Sunand T. Joshi (ed.), H. P. Lovecraft: Four Decades of Criticism, Athens (OH), Ohio University Press, 1980, p. 50-62.

Maurice Lévy, Lovecraft ou du fantastique, Paris, Union Générale d'Éditions, 1972.

Edward Lipiński, Dieux et déesses de l'univers phénicien et punique, Leuven, Peeters, 1995.

Giuseppe Lippi, "Note alla Parte quinta", in Howard Phillips Lovecraft, Lettere dall'altrove. Epistolario 1915-1937, a cura di Id., Milano, Mondadori, 1993, p. 361-367.

Howard Phillips Lovecraft, Colui che sussurrava nel buio, traduzione di Sarah Cantoni, in Id., Colui che sussurrava nel buio, a cura di Carlo Fruttero, Coll. "Urania”, n. 310, Milano, Mondadori, 1963, p. 4-62.

Howard Phillips Lovecraft, Selected Letters I: 1911-1924, Edited by August Derleth, Donald Wandrei, Sauk City (WI), Arkham House, 1965. 
Howard Phillips Lovecraft, Selected Letters V: 1934-1937, Edited by August Derleth, James Turner, Sauk City (WI), Arkham House, 1976.

Howard Phillips Lovecraft, L'orrore soprannaturale nella letteratura, traduzione di Alda Carrer, in Id., Opere complete, a cura di Giuseppe Lippi, Milano, SugarCo, 1983 [1973], p. 15-66.

Howard Phillips Lovecraft, Opere complete, a cura di Giuseppe Lippi, Milano, SugarCo, 1983 [1973]. Howard Phillips Lovecraft, Lettere dall'altrove. Epistolario 1915-1937, a cura di Giuseppe Lippi, Milano, Mondadori, 1993.

Howard Phillips Lovecraft, L'orrore della realtà. La visione del mondo del rinnovatore della narrativa fantastica. Lettere 1915-1937, a cura di Gianfranco de Turris, Sebastiano Fusco, traduzione di Massimo Berruti, Roma, Edizioni Mediterranee, 2007.

Howard Phillips Lovecraft, The Horror in the Museum and Other Revisions, Introduction by Stephen Jones, New York, Ballantine Books, 2007.

Howard Phillips Lovecraft, The Complete Fiction, Introduction by Sunand T. Joshi, New York, Barnes \& Noble, 2011.

Howard Phillips Lovecraft, Tutti i romanzi e i racconti. L'incubo - Il sogno - Il mito. Le storie del Ciclo di Cthulhu, Miscellanea e Saggi, a cura di Gianni Pilo, Sebastiano Fusco, Roma, Newton Compton, 2016.

Arthur Machen, Il Grande Dio Pan, traduzione, note e appunti di Alessandro Zabini, Roma, Tre Editori, 2016.

Steven J. Mariconda, "Lovecraft's Cosmic Imagery”, in Sunand T. Joshi, David E. Schultz (eds.), An Epicure in the Terrible: A Centennial Anthology of Essays in Honor of H. P. Lovecraft, Cranbury (NJ), Associated University Presses, 1991, p. 188-198.

Gilles Menegaldo, “Gothic Convention and Modernity in John Ramsey Campbell's Short Fiction”, in Victor Sage, Allan Lloyd Smith (eds.), Modern Gothic: A Reader, Manchester, Manchester University Press, 1996, p. 188-197.

Nadia Minerva, "La luna e il diavolo. Dalla demonologia classica al mito di Lilith nel Romanticismo francese", in Id. (a cura di), La luna allo specchio. Rappresentazioni, simbologie e metafore seleniche nella letteratura e nell'immaginario, Bologna, Pàtron Editore, 1990, p. 35-53.

Dirk W. Mosig, "Lovecraft mitografo", in August Derleth (a cura di), I Miti di Cthulhu, introduzione, prologo, parte prima, epilogo, bibliografia, note e commenti di Gianfranco de Turris, Sebastiano Fusco, Roma, Fanucci, 1975, p. 23-31.

Will Murray, "On the Natures of Nug and Yeb", in Lovecraft Studies, n. 9, 1984, p. 52-59.

Erich Neumann, "La luna e la coscienza matriarcale", in Id., La psicologia del femminile, Roma, Astrolabio-Ubaldini, 1975, p. 46-77.

Erich Neumann, Storia delle origini della coscienza, Roma, Astrolabio-Ubaldini, 1978.

Erich Neumann, La Grande Madre. Fenomenologia delle configurazioni femminili dell'inconscio, Roma, Astrolabio-Ubaldini, 1981.

Ulf Oldenburg, The Conflict Between El and Baal in Canaanite Religion, Leiden, Brill, 1969.

Anthony Pearsall, "Shub-Niggurath", in Id., The Lovecraft Lexicon: A Reader's Guide to Persons, Places and Things in the Tales of H. P. Lovecraft, Las Vegas (NV), New Falcon Publications, 2005, p. 457-458.

Peter Penzoldt, The Supernatural in Fiction, New York, Humanities Press, 1965.

Gianni Pilo, "Revisioni e collaborazioni di H. P. Lovecraft", in Howard Phillips Lovecraft, Tutti i romanzi e i racconti. L'incubo - Il sogno - Il mito. Le storie del Ciclo di Cthulhu, Miscellanea e Saggi, a cura di Gianni Pilo, Sebastiano Fusco, Roma, Newton Compton, 2016, p. 28-29.

Robert M. Price, "Lovecraft's Concept of Blasphemy", in Id., H. P. Lovecraft and the Cthulhu Mythos, Mercer Island (WA), Starmont House, 1990, p. 3-11 [prev. publ. in Crypt of Cthulhu, n. 1, 1981, p. 3-15]. Robert M. Price, "The Lovecraft-Derleth Connection", in Id., H. P. Lovecraft and the Cthulhu Mythos, Mercer Island (WA), Starmont House, 1990, p. 96-102 [prev. publ. in Crypt of Cthulhu, n. 6, 1982, p. 3-8]. Robert M. Price, "The Revision Mythos", in Id., H. P. Lovecraft and the Cthulhu Mythos, Mercer Island (WA), Starmont House, 1990, p. 103-112 [prev. publ. in Crypt of Cthulbu, n. 11,1983, p. 15-19, and repr. rev. in Lovecraft Studies, n. 4, 1985, p. 43-50]. 
Robert M. Price, "Mythos Names and How to Say Them", in Id., H. P. Lovecraft and the Cthulhu Mythos, Mercer Island (WA), Starmont House, 1990, p. 113-121 [prev. publ. as "Cthul-who? How do you Pronounce «Cthulhu»?", in Crypt of Cthulhu, n. 9, 1982, p. 3, and repr. rev. in Lovecraft Studies, n. 6, 1987, p. 47-53].

Robert M. Price, "A Lovecraftian Taxonomy", in Id., H. P. Lovecraft and the Cthulbu Mythos, Mercer Island (WA), Starmont House, 1990, p. 128-131 [prev. publ. in Crypt of Cthulhu, n. 12, 1983, p. 17-19]. Robert M. Price, "Lovecraft's «Artificial Mythology»", in Sunand T. Joshi, David E. Schultz (eds.), An Epicure in the Terrible: A Centennial Anthology of Essays in Honor of H. P. Lovecraft, Cranbury (NJ), Associated University Presses, 1991, p. 247-256.

Robert M. Price, "Introduction", in Id. (sel. and ed.), The Shub-Niggurath Cycle: Tales of the Black Goat with a Thousand Young, Oakland (CA), Chaosium, 1994, p. XI-XVI.

Jean Przyluski, La Grande Déesse. Introduction à l'étude comparative des religions, Paris, Payot, 1950.

David Punter, Storia della letteratura del terrore: il «gotico» dal Settecento ad oggi, sec. ed. riveduta e ampliata, Roma, Editori Riuniti, 2000.

David Punter (ed.), A New Companion to the Gothic, Malden (MA), Wiley-Blackwell, 2012.

Nicholas Royle, "Campbell, Ramsey", in William Hughes, David Punter, Andrew Smith (eds.), The Encyclopedia of the Gothic, Malden (MA), Wiley, 2016, p. 105-106.

Maximilian J. Rudwin, The Devil in Legend and Literature, Chicago, Open Court Publishing Company, 1931. Jeffrey B. Russell, The Devil: Perceptions of Evil from Antiquity to Primitive Christianity, Ithaca and London, Cornell University Press, 1977.

Victor Sage, Allan Lloyd Smith (eds.), Modern Gothic: A Reader, Manchester, Manchester University Press, 1996.

Darrell Schweitzer (ed.), Discovering Modern Horror Fiction, Berkeley Heights (NJ), Wildside Press, 1999, 2 voll.

Philip A. Shreffler, The H. P. Lovecraft Companion, Westport (CT), Greenwood Press, 1977.

Mary Ellen Snodgrass, "Lovecraft, H. P.", in Id., Encyclopedia of Gothic Literature, New York, Facts on File, 2005, p. 214-215.

Barton Levi St. Armand, The Roots of Horror in the Fiction of H. P. Lovecraft, Elizabethtown (NY), Dragon Press, 1977.

Richard L. Tierney, "The Derleth Mythos", in Meade Frierson III, Penny Frierson (eds.), HPL: A Tribute to Howard Phillips Lovecraft, Birmingham (AL), The Editors, 1972, p. 53.

Richard L. Tierney, "Lovecraft and the Cosmic Quality in Fiction", in Sunand T.Joshi (ed.), H. P. Lovecraft: Four Decades of Criticism, Athens (OH), Ohio University Press, 1980, p. 191-195.

Juliette Vion-Dury, "Cthulhu", in Pierre Brunel, Juliette Vion-Dury (dir.), Dictionnaire des mythes du fantastique, Limoges, Pulim, 2003, p. 51-90.

Elémire Zolla (a cura di), I contemporanei: Novecento americano, Roma, Lucarini, 1986, 3 voll.

\section{Note}

1. David Punter classifica Lovecraft nel "tardo «gotico» americano", segnalando tuttavia che la sua opera conserva almeno due motivi propri del modello gotico tradizionale: la paura del passato - la quale non esclude l'attrazione che questo esercita - e il gusto iniquo per una conoscenza proibita (cfr. David Punter, Storia della letteratura del terrore: il «gotico» dal Settecento ad oggi, sec. ed. riveduta e ampliata, Roma, Editori Riuniti, 2000, p. 240, p. 252-254). Sull'allineamento tematico e stilistico di Lovecraft con il modello gotico, si veda inoltre Mary Ellen Snodgrass, "Lovecraft, H. P.", in Id., Encyclopedia of Gothic Literature, New York, Facts on File, 2005, p. 214-215.

2. Secondo la visione cosmicistica maturata da Lovecraft - esposta soprattutto nel suo epistolario, di valore letterario non inferiore a quello della sua narrativa - l'universo consiste in un insieme di "forze cieche che agiscono secondo schemi fissi ed eterni" (Howard Phillips Lovecraft, L'orrore della realtà. La visione del mondo del rinnovatore della narrativa fantastica. Lettere 1915-1937, a cura di Gianfranco de 
Turris, Sebastiano Fusco, traduzione di Massimo Berruti, Roma, Edizioni Mediterranee, 2007, p. 84), in cui l'umanità non è che un "banale incidente" (Howard Phillips Lovecraft, Selected Letters I: 1911-1924, Edited by August Derleth, Donald Wandrei, Sauk City (WI), Arkham House, 1965, p. 56). Per un orientamento critico sul cosmicismo lovecraftiano, si consultino Richard L. Tierney, "Lovecraft and the Cosmic Quality in Fiction", in Sunand T. Joshi (ed.), H. P. Lovecraft: Four Decades of Criticism, Athens (OH), Ohio University Press, 1980, p. 191-195; Steven J. Mariconda, "Lovecraft's Cosmic Imagery", in Sunand T. Joshi, David E. Schultz (eds.), An Epicure in the Terrible: A Centennial Anthology of Essays in Honor of H. P. Lovecraft, Cranbury (NJ), Associated University Presses, 1991, p. 188-198; Sunand T. Joshi, A Dreamer and a Visionary: H. P. Lovecraft in His Time, Liverpool, Liverpool University Press, 2001, p. 132 ss., p. 181-182.

3. Barton Levi St. Armand, The Roots of Horror in the Fiction of H. P. Lovecraft, Elizabethtown (NY), Dragon Press, 1977, p. 36.

4. Ibid.

5. Ibid. Anche Philip A. Shreffler, nella sua magistrale monografia sul solitario di Providence, osserva come, nonostante nella narrativa lovecraftiana non manchino chiari segni della sua familiarità con le convenzioni gotiche - si pensi ai "castelli fatiscenti" e soprattutto ai "villaggi in rovina" (Philip A. Shreffler, The H. P. Lovecraft Companion, Westport (CT), Greenwood Press, 1977, p. 6) -, l'autore angloamericano "ebbe la capacità di rifuggirlo, o almeno di modificarlo per i propri intenti, eliminandone i clichés” (ibid., p. 18).

6. Ritenuto il primo - e il più significativo - saggio scritto da un "praticante" del genere indagato (cfr. Clive Bloom, "Horror Fiction: In Search of a Definition", in David Punter (ed.), A New Companion to the Gothic, Malden (MA), Wiley-Blackwell, 2012, p. 214), Supernatural Horror in Literature consacra Lovecraft a critico della letteratura, testimoniando come "il suo approccio [...] alla weird fiction era tanto quello dello studioso quanto quello dell'artista creativo" (Peter Penzoldt, The Supernatural in Fiction, New York, Humanities Press, 1965, p. 165).

7. Cfr. Shreffler, The H. P. Lovecraft Companion, p. 7. Si consulti, in merito, Howard Phillips Lovecraft, L'orrore soprannaturale nella letteratura, traduzione di Alda Carrer, in Id., Opere complete, a cura di Giuseppe Lippi, Milano, SugarCo, 1983 [1973], p. 41 ss.

8. Lovecraft, L'orrore soprannaturale, p. 18.

9. Ibid., p. 19.

10. Shreffler, The H. P. Lovecraft Companion, p. 9.

11. Ibid., p. 155.

12. Cfr. ibid., p. 9; Maurice Lévy, Lovecraft ou du fantastique, Paris, Union Générale d'Éditions, 1972, p. 74-75; Dirk W. Mosig, "Lovecraft mitografo", in August Derleth (a cura di), I Miti di Cthulhu, introduzione, prologo, parte prima, epilogo, bibliografia, note e commenti di Gianfranco de Turris, Sebastiano Fusco, Roma, Fanucci, 1975, p. 25-27. Tale prospettiva metafisica traccia, tra l'altro, una cesura essenziale tra Lovecraft ed Edgar Allan Poe - da lui definito il suo "Dio della Narrativa" (Lovecraft, Selected Letters I, p. 20) e a cui dedica un'intera sezione di Supernatural Horror in Literature (p. 37-41) - il quale piuttosto "usa la paura nei limiti di una psicologia in cui si riflettono i suoi fantasmi" (Francis Lacassin, "Lovecraft et les trous de la toile peinte", in Cabiers de l'Herne, n. 12, 1969, p. 109). Come rileva efficacemente Punter, "mentre Poe ricollega la paura alla «vita interiore», Lovecraft è assolutamente privo di interesse psicologico, i suoi terrori sono in tutto e per tutto quelli provenienti da un di fuori incomprensibile, quelli dell'individuo preso nella morsa dell'invadenza estranea" (Storia, p. 252).

13. Shreffler, The H. P. Lovecraft Companion, p. 155. In merito, cfr. inoltre Fritz Leiber Jr., "A Literary Copernicus", in Joshi (ed.), H. P. Lovecraft, spec. p. 50-52.

14. St. Armand, The Roots, p. 4.

15. Cfr. Mosig, "Lovecraft mitografo", p. 27; Franco La Polla, "Lovecraft, il gotico e i segni cosmici", in Il Verri, n. 37,1971, p. 73. Sulla nozione di Outer Gods e sulla problematica classificatoria relativa a questi e ai Great Old Ones, si consultino Daniel Harms, "Great Old Ones", in Id., Encyclopedia Cthulhiana, Oakland (CA), Chaosium, 1994, p. 86-88; Daniel Harms, "Outer Gods”, in Id., Encyclopedia, p. 
161-162; Robert M. Price, "A Lovecraftian Taxonomy", in Id., H. P. Lovecraft and the Cthulhu Mythos, Mercer Island (WA), Starmont House, 1990, p. 128-131.

16. Shreffler, The H. P. Lovecraft Companion, p. 155.

17. Cfr. Mosig, "Lovecraft mitografo", p. 26.

18. Lin Carter, Lovecraft: A Look Behind the "Cthulhu Mythos», New York, Ballantine Books, 1972, p. 20. La critica è stata (mal) indirizzata a denominare la mythopoeia di Lovecraft "Cthulhu Mythos" - ossia "Mito di Cthulhu" talvolta erroneamente reso al plurale, "Miti di Cthulhu" - da parte di August Derleth (in proposito, si veda Richard L. Tierney, "The Derleth Mythos", in Meade Frierson III, Penny Frierson (eds.), HPL: A Tribute to Howard Phillips Lovecraft, Birmingham (AL), The Editors, 1972, p. 53; si veda, tuttavia, Robert M. Price, “The Lovecraft-Derleth Connection”, in Id., H. P. Lovecraft, p. 96-102); in realtà, il maestro di Providence non ha mai utilizzato questa terminologia, optando piuttosto per i derivati Cthulhuism e Yog-Sothothery, come nella lettera ad August Derleth datata 16 maggio 1931 (cit. da Sunand T. Joshi, David E. Schultz, "Cthulhu Mythos", in Id., An H. P. Lovecraft Encyclopedia, Westport (CT), Greenwood Press, 2001, p. 52). Dirk W. Mosig ritiene ben più appropriata, in merito, la formula "Ciclo Mitico di Yog-Sothoth" (Mosig, "Lovecraft mitografo", p. 25). Comunque lo si nomini, il Mito fondato da Lovecraft non deve intendersi come un corpus di racconti, bensì come una "struttura portante che sta dietro a gran parte della [sua] opera più famosa” (Punter, Storia, p. 252).

19. Cfr. Carl Gustav Jung, Gli archetipi dell'inconscio collettivo, Torino, Bollati Boringhieri, 2011, p. 18.

20. Gilbert Durand, "Méthode archétypologique: de la mythocritique à la mythanalyse", in Id., Champs de l'imaginaire, textes réunis par Danièle Chauvin, Grenoble, Ellug, Université Stendhal, 1996, p. 141.

21. Shreffler, The H. P. Lovecraft Companion, p. 155. Sugli ostacoli che la mythopoeia lovecraftiana pone al principio stesso di rappresentabilità letteraria e a ogni possibilità di dissezione critica, si veda Armando Gnisci, "Howard Phillips Lovecraft", in Elémire Zolla (a cura di), I contemporanei: Novecento americano, Roma, Lucarini, 1986, vol. I, p. 556-558.

22. Lévy, Lovecraft, p. 17.

23. Se è fuor di dubbio corretto denominare il monumento immaginario eretto da Lovecraft "pseudomitologia" (Mosig, "Lovecraft mitografo", p. 26; si veda anche Joshi, A Dreamer, p. 244), sarà altrettanto appropriato definire, piuttosto, "pseudoteologia" quel nozionismo che concerne un soggetto divino come Shub-Niggurath, il cui nome è molto familiare al lettore per la sua martellante ricorrenza nella "litania lovecraftiana", ma di cui non si contemplano degli autentici mythoi (a riguardo, cfr. Robert M. Price, "Introduction", in Id. (sel. and ed.), The Shub-Niggurath Cycle: Tales of the Black Goat with a Thousand Young, Oakland (CA), Chaosium, 1994, p. XI-XII).

24. Lin Carter, Lovecraft, p. 173.

25. Daniel Harms, "Shub-Niggurath", in Id., Encyclopedia, p. 192.

26. Nella ricostruzione proposta da Shreffler, Shub-Niggurath si trova immediatamente dopo gli onnipotenti e onnipresenti Yog-Sothoth - di cui figura talvolta come moglie - e Azathoth (cfr. Shreffler, The H. P. Lovecraft Companion, p. 158-159). Tuttavia, nella lettera di Lovecraft a James F. Morton datata 27 aprile 1933 - in cui il visionario di Providence traccia un fantasmatico albero genealogico (non necessariamente sovrapponibile a una gerarchia) che illustrerebbe la sua discendenza dagli stessi "demoni" di sua invenzione - Shub-Niggurath si trova, accanto al consorte Yog-Sothoth, più in basso rispetto alla vetta Azathoth nonché rispetto a Nyarlathotep (cfr. Howard Phillips Lovecraft, Lettere dall'altrove. Epistolario 1915-1937, a cura di Giuseppe Lippi, Milano, Mondadori, 1993, p. 255). Altri studiosi relegano Shub-Niggurath a una posizione marginale nella mythopoeia di Lovecraft, trattandolo non solo dopo gli dèi di cui sopra, ma persino dopo le entità extraterrestri quali Cthulhu e Dagon (cfr. Lévy, Lovecraft, p. 124-125), di natura non accertatamente divina (cfr. Shreffler, The H. P. Lovecraft Companion, p. 160).

27. Lovecraft, Lettere, p. 333.

28. Cfr. August Derleth, "Le «revisioni» di Lovecraft", in Howard Phillips Lovecraft, Nelle spire di Medusa, traduzione integrale di Roberta Rambelli, Roma, Fanucci, 1976, p. 17. Per una classificazione delle revisioni, si veda Gianni Pilo, "Revisioni e collaborazioni di H. P. Lovecraft", in Howard Phillips 
Lovecraft, Tutti i romanzi e i racconti. L'incubo - Il sogno - Il mito. Le storie del Ciclo di Cthulhu, Miscellanea e Saggi, a cura di Gianni Pilo, Sebastiano Fusco, Roma, Newton Compton, 2016, p. 28-29.

29. Su questa classificazione, e sul caso limite di Shub-Niggurath nel Revision Pantheon, si veda Robert M. Price, "The Revision Mythos", in Id., H. P. Lovecraft, spec. p. 103-106.

30. Cfr. Howard Phillips Lovecraft, Tutti i romanzi e i racconti, p. 375. La traduzione italiana del titolo del racconto è, qui, L'ultimo esperimento di Clarendon. D'ora in avanti, nel presente saggio si indicherà per ogni opera di Lovecraft l'anno di pubblicazione e non quello di redazione.

31. Cfr. ibid., p. 1598. La traduzione italiana del titolo del racconto è, qui, Medusa.

32. Cfr. ibid., p. 1336.

33. Cfr. ibid., p. 1297.

34. Cfr. ibid., p. 1371. Abbiamo integrato la scelta traduttiva di Gianni Pilo, per rispettare maggiormente il testo originale, nonché la connotazione di Shub-Niggurath (Howard Phillips Lovecraft, The Horror in the Museum and Other Revisions, Introduction by Stephen Jones, New York, Ballantine Books, 2007, p. 144: "the All-Mother").

35. Cfr. Lovecraft, Tutti i romanzi e i racconti, p. 1371.

36. Cfr. ibid., p. 1700. La traduzione italiana del titolo del racconto è, qui, Dai millenni.

37. Cfr. ibid., p. 1645.

38. Cfr. ibid., p. 1680, p. 1685.

39. Cfr. ibid., p. 1781.

40. Cfr. ibid., p. 1634.

41. Cfr. Shreffler, The H. P. Lovecraft Companion, p. 106; Price, "Introduction”, p. XI.

42. L'articolo determinativo "il" precede sempre l'epiteto e pertanto potrebbe considerarsi parte di esso, ma (contrariamente a quanto afferma Rodolfo A. Ferraresi, "The Question of Shub-Niggurath", in Crypt of Cthulhu, n. 35, 1985, p. 18) non è sempre a lettera maiuscola (Howard Phillips Lovecraft, The Complete Fiction, Introduction by Sunand T. Joshi, New York, Barnes \& Noble, 2011, p. 683: "the Black Goat of the Woods"). Probabilmente lo è quasi sempre perché il più delle volte segue al punto esclamativo.

43. Cfr. Robert M. Price, "Mythos Names and How to Say Them”, in Id., H. P. Lovecraft, p. 117; Shreffler, The H. P. Lovecraft Companion, p. 170.

44. Cfr. Lovecraft, Tutti i romanzi e $i$ racconti, p. 1401-1402. È l'unico caso, nell'intera edizione di riferimento, in cui il traduttore Gianni Pilo, apparentemente senza una ragione, opta per la resa di "Thousand Young" con "Prole Innumerevole", anziché con "Mille Cuccioli". È tra l'altro curioso che, nella prima traduzione italiana in assoluto di The Whisperer in Darkness da parte di Sarah Cantoni (in seguito riproposta nell'edizione delle opere complete di Lovecraft a cura di Lippi per SugarCo), sono da segnalare, proprio in questi contesti, delle omissioni parziali nella resa italiana dell'epiteto di ShubNiggurath - nella fattispecie, non viene riportato l'equivalente italiano di "with a Thousand Young" - e addirittura una lacuna là dove dovrebbe figurare una delle invocazioni rivolte alla divinità (Howard Phillips Lovecraft, Colui che sussurrava nel buio, traduzione di Sarah Cantoni, in Id., Colui che sussurrava nel buio, a cura di Carlo Fruttero, Coll. "Urania”, n. 310, Milano, Mondadori, 1963, p. 22-23; Lovecraft, Opere complete, p. 452). All'incompletezza e inesattezza delle prime traduzioni italiane di Lovecraft si accenna in Gianfranco de Turris, Sebastiano Fusco, Howard Phillips Lovecraft, Firenze, La Nuova Italia, 1979, p. 125.

45. Cfr. Lovecraft, Tutti i romanzi e $i$ racconti, p. 1666. In questo racconto, vi è anche un'occorrenza dell'esclamazione semplice "Iä! Shub-Niggurath!" (ibid.,p. 1659; nella traduzione italiana non figura il consueto punto esclamativo, invece presente nella versione originale; cfr. Lovecraft, The Complete Fiction, p. 927).

46. Cfr. ancora Lovecraft, Tutti i romanzi e i racconti, p. 1401-1402.

47. Cfr. ibid., p. 1401.

48. Un primo sospetto, in verità, viene instillato da un indizio che trascende e precede gli epiteti di invenzione lovecraftiana: la sessualità maschile di un dio di invenzione dell'irlandese Lord Dunsany (che Lovecraft indica come uno dei suoi maestri; cfr. Lovecraft, L'orrore soprannaturale, p. 61-62), 
menzionato nel racconto Idle Days on the Yann (Giorni d'ozio sullo Yann, 1910), Sheol Nugganoth, il cui nome avrebbe ispirato quello di Shub-Niggurath (cfr. Price, "Mythos Names", p. 117).

49. Lin Carter, Lovecraft, p. 71. Si tratta di esordio se si esclude la revisione per de Castro, The Last Test (1928); è a ogni effetto un "esordio" nella narrativa esclusiva di Lovecraft.

50. Shreffler, The H. P. Lovecraft Companion, p. 106.

51. Ibid., p. 159.

52. De Turris, Fusco, Howard Phillips Lovecraft, p. 61.

53. Shreffler, The H. P. Lovecraft Companion, p. 159. Più di recente, anche Juliette Vion-Dury, nella sua guida al Ciclo Mitico di Lovecraft, manifesta un margine di incertezza sulla femminilità di ShubNiggurath, descrivendola come 1'"unica divinità femminile, o che è piuttosto legata al femminile [ou procédant quelque peu du féminin], d[el] pantheon [lovecraftiano]" (Juliette Vion-Dury, "Cthulhu", in Pierre Brunel, Juliette Vion-Dury (dir.), Dictionnaire des mythes du fantastique, Limoges, Pulim, 2003, p. 57.

54. Price, "The Revision Mythos", p. 106. Si veda anche Robert M. Price, "Lovecraft's "Artificial Mythology»", in Sunand T. Joshi, David E. Schultz (eds.), An Epicure, p. 252.

55. Price, "Mythos Names", p. 117. Cfr. anche Ferraresi, "The Question”, p. 17.

56. Price, "Introduction", p.X.

57. Lovecraft, Lettere, p. 333. Si tratta della lettera a Kuttner del 1936 in cui Shub-Niggurath è demonefemmina, moglie e madre. Il seguito - in traduzione di Lippi - è: "Il suo culto - che si riassume nel rito simbolico della Capra dai Mille Cuccioli - è una delle più orrende tradizioni ereditate dalla razza umana dai tempi preumani" (ibid.).

58. Giuseppe Lippi, “Note alla Parte quinta”, in Lovecraft, Lettere, p. 365. In proposito, cfr. anche Gavin Callaghan, H. P. Lovecraft's Dark Arcadia: The Satire, Symbology and Contradiction, Jefferson (NC) and London, McFarland \& Company, 2013, p. 162-163.

59. Lovecraft, Tutti i romanzi e i racconti, p. 1700. La nostra integrazione rispetta maggiormente il testo originale (Lovecraft, The Horror, p. 273: "copper temple").

60. Limitiamo l'osservazione alla narrativa di Lovecraft, poiché nel suo epistolario vi è in realtà un secondo caso in cui Shub-Niggurath e il Capro sono disgiunti: la lettera a Willis Conover datata 1 settembre 1936, nella quale la divinità è definita un'entità infernale dalle sembianze di nuvola [...], in onore della quale culti senza nome celebrano il rito del Capro dai Mille Cuccioli" (Howard Phillips Lovecraft, Selected Letters V: 1934-1937, Edited by August Derleth, James Turner, Sauk City (WI), Arkham House, 1976, p. 303). Ci riserviamo di affrontare la caratterizzazione nubiforme di ShubNiggurath in un futuro studio.

61. Ferraresi, "The Question”, p. 17-18.

62. Will Murray, "On the Natures of Nug and Yeb”, in Lovecraft Studies, n. 9, 1984, p. 58.

63. Erich Neumann, La Grande Madre. Fenomenologia delle configurazion i femminili dell'inconscio, Roma, Astrolabio-Ubaldini, 1981, p. 63.

64. Su Astarte come dea lunare, si vedano Alexander Haggerty Krappe, La genèse des mythes, Paris, Payot, 1938, p. 104; Edward Lipiński, Dieux et déesses de l'univers phénicien et punique, Leuven, Peeters, 1995, p. 153-154. Si consultino anche le fonti indicate da Nadia Minerva, "La luna e il diavolo. Dalla demonologia classica al mito di Lilith nel Romanticismo francese", in Id. (a cura di), La luna allo specchio. Rappresentazioni, simbologie e metafore seleniche nella letteratura e nell'immaginario, Bologna, Pàtron Editore, 1990, p. 45-46. Come rileva Callaghan, la luna quale archetipo femminile occupa un posto rilevante nella poesia di Lovecraft - in cui, talvolta, si manifesta proprio nella forma divina di Astarte (cfr. Callaghan, H. P. Lovecraft's Dark Arcadia, p. 124; cfr. anche p. 136-137).

65. Cfr. Jean Przyluski, La Grande Déesse. Introduction à l'étude comparative des religions, Paris, Payot, 1950, p. 134-135.

66. Jean Chevalier, Alain Gheerbrant, Dizionario dei simboli, Milano, BUR, 2011, vol. II, p. 48.

67. Mircea Eliade, Trattato di storia delle religioni, Torino, Bollati Boringhieri, 2008, p. 149.

68. Ibid., p. 148. 
Valentina Sirangelo

69. Ibid.

70. Ibid.

71. Cfr. Ulf Oldenburg, The Conflict Between El and Baal in Canaanite Religion, Leiden, Brill, 1969, p. 44.

72. Izak Cornelius, The Many Faces of the Goddess: The Iconography of the Syro-Palestinian Goddesses Anat, Astarte, Qedeshet, and Asherah c. 1500-1000 BCE, Fribourg, Academic Press, and Göttingen, Vandenhoeck \& Ruprecht, 2004, p. 34.

73. Anne Baring, Jules Cashford, The Myth of the Goddess. Evolution of an Image, London, Penguin, 1993, p. 147.

74. Erich Neumann, "La luna e la coscienza matriarcale", in Id., La psicologia del femminile, Roma, Astrolabio-Ubaldini, 1975, p. 48.

75. Sul dio figlio-amante, si veda Erich Neumann, Storia delle origini della coscienza, Roma, AstrolabioUbaldini, 1978, p. 60 ss.

76. Gilbert Durand, Le strutture antropologiche dell'immaginario. Introduzione all'archetipologia generale, Bari, Edizioni Dedalo, 2009, p. 357-360.

77. Esiste un'altra divinità maschile, appartenente al Revision Pantheon lovecraftiano, con cui ShubNiggurath, in un'occasione, sembra addirittura fondersi più che confondersi: il Dio-Serpente Yig. Nella revisione per De Castro The Electric Executioner (Il boia elettrico, 1930) figura infatti un'entità denominata "Niggurat[1]-Yig" (Lovecraft, Tutti $i$ romanzi e i racconti, p. 397). La nostra integrazione rispetta maggiormente il nome nel testo originale (Lovecraft, The Horror, p. 74: "Niguratl-Yig", che tuttavia non presenta la consueta geminazione consonantica - in merito, cfr. Price, "The Revision Mythos", p. 107, p. 111). Ci proponiamo di approfondire il rapporto tra Shub-Niggurath e Yig in un futuro studio.

78. Ferraresi, "The Question”, p. 22. A riguardo, cfr. anche Oldenburg, The Conflict, p. 44.

79. Analogamente, lo storico Renzo Giorgetti, pur avendo tracciato proficuamente un nesso tra la Dea e il Capro attraverso un parallelo con la mitologia basca - recando l'esempio della dea lunare Mari che può manifestarsi sotto forma del dio-capro Akerbeltz, suo paredro (cfr. Renzo Giorgetti, Archetipi lovecraftiani: l'Eterno Femminile, Stienta, Edizioni Diversa Sintonia, 2012, p. 70-71) - ha mancato di motivare la mutevolezza sessuale di Shub-Niggurath sulla base dell'archetipologia lunare stessa.

80. Ferraresi, "The Question”, p. 22.

81. Cfr. Lovecraft, L'orrore soprannaturale, p. 55-59.

82. Arthur Machen, Il Grande Dio Pan, traduzione, note e appunti di Alessandro Zabini, Roma, Tre Editori, 2016, p. 102.

83. Chevalier, Gheerbrant, Dizionario, vol. II, p. 181.

84. Ibid.

85. James G. Frazer, Il ramo d'oro. Studio sulla magia e sulla religione, Roma, Newton Compton, 2006, p. 525-526.

86. Durand, Le strutture, p. 170.

87. In parte, ciò è riconosciuto in Shreffler, The H. P. Companion, p. 159.

88. Eliade, Trattato, p. 86.

89. Per un orientamento, si consulti Chris Jarocha-Ernst, A Cthulhu Mythos Bibliography and Concordance, Seattle (WA), Armitage House, 1999, p. 368.

90. Gary William Crawford, "Urban Gothic: The Fiction of Ramsey Campbell”, in Darrell Schweitzer (ed.), Discovering Modern Horror Fiction, Berkeley Heights (NJ), Wildside Press, 1999, vol. I, p. 14. A riguardo, si veda anche Nicholas Royle, "Campbell, Ramsey", in William Hughes, David Punter, Andrew Smith (eds.), The Encyclopedia of the Gothic, Malden (MA), Wiley, 2016, p. 105. A partire dalla fine degli anni Sessanta, quando si distacca dall'egida lovecraftiana, la narrativa di Campbell diviene propriamente neo-gotica da un punto di vista sia stilistico che tematico (cfr. Crawford, "Urban Gothic", p. 15-17). Sulle integrazioni di Campbell al Ciclo Mitico lovecraftiano, si veda August Derleth, "Genesi e struttura dei «Miti di Cthulhu»", in Id. (a cura di), I Miti di Cthulhu, introduzione, prologo, parte prima, epilogo, bibliografia, note e commenti di Gianfranco de Turris, Sebastiano Fusco, Roma, Fanucci, 1975, p. 18-19. 
91. La prima edizione della raccolta fu pubblicata dalla Arkham House, la casa editrice co-fondata da due discepoli di Lovecraft, August Derleth e Donald Wandrei (cfr. J. Ramsey Campbell, The Inhabitant of the Lake and Less Welcome Tenants, Sauk City, Arkham House, 1964; a riguardo, si consulti anche Ramsey Campbell, Stefan R. Dziemianowicz, Sunand T. Joshi, The Core of Ramsey Campbell: A Bibliography and Reader's Guide, West Warwick (RI), Necronomicon Press, 1995, p. 9). Fu poi pubblicata nuovamente decenni dopo: nell'edizione più recente, il titolo è leggermente differente - in particolare, "Less Welcome Tenants" è sostituito da "Other Unwelcome Tenants" ("altri inquilini indesiderati") - e il nome dell'autore non è più preceduto dalla "J." (Ramsey Campbell, The Inbabitant of the Lake and Other Unwelcome Tenants, Hornsea, PS Publishing, 2011). Per le citazioni dal racconto The Moon-Lens, ci riferiremo tuttavia, nel presente studio, al volume antologico curato da Price che lo include (The ShubNiggurath Cycle), in traduzione nostra.

92. Cfr. Stefan Dziemianowicz, "Mastering Darkness: An Interview with Ramsey Campbell", in Gary William Crawford (ed.), Ramsey Campbell: Critical Essays on the Modern Master of Horror, Lanham (MD), Scarecrow Press, 2014, p. 118.

93. Gilles Menegaldo, "Gothic Convention and Modernity in John Ramsey Campbell's Short Fiction", in Victor Sage, Allan Lloyd Smith (eds.), Modern Gothic: A Reader, Manchester, Manchester University Press, 1996, p. 188. A riguardo, si veda soprattutto Sunand T. Joshi, Ramsey Campbell and Modern Horror Fiction, Liverpool, Liverpool University Press, 2001, p. 43 ss.

94. Sulla topografia fantastica del primo Campbell, si consultino Carter, Lovecraft, p. 173; Ramsey Campbell, "A Word from the Author", in Id., The Inhabitant of the Lake and Other Unwelcome Tenants, p. VII-IX.

95. Lévy, Lovecraft, p. 66.

96. Lidea di una porta collinare il cui meccanismo di apertura impiega il riflesso lunare deriva da un'annotazione frammentaria a carattere visionario di Lovecraft del 1931: "Un'antica necropoli. Sul fianco di una collina, una porta di bronzo si apre quando la luce lunare la colpisce. Ma deve essere focalizzata da una lente posta in cima ad una colonna antistante" (Lovecraft, Tutti i romanzi e i racconti, p. 743).

97. Cfr. Eliade, Trattato, p. 155.

98. Sul motivo lovecraftiano del culto sotto mentite spoglie - relativamente però alla sua divinità Nyarlathotep - si veda Shreffler, The H. P. Lovecraft Companion, p. 159 ss.

99. Ramsey Campbell, The Moon-Lens, in Price (sel. and ed.), The Shub-Niggurath Cycle, p. 47. Il lungo profilo storico-religioso correla Shub-Niggurath non solo al Pan del mito classico, ma anche al Satana del mito cristiano, definito da Ferraresi "la metamorfosi medievale di Pan" (Ferraresi, "The Question", p. 18; in merito, si veda anche Jeffrey B. Russell, The Devil: Perceptions of Evil from Antiquity to Primitive Christianity, Ithaca and London, Cornell University Press, 1977, p. 126, p. 254) e da lui segnalato come altamente incisivo nell'elaborazione del "malevolo" Shub-Niggurath già da parte di Lovecraft. Sui tratti caprini del demonio, si vedano ad esempio Maximilian J. Rudwin, The Devil in Legend and Literature, Chicago, Open Court Publishing Company, 1931, p. 39 ss.; Germain Bazin, "Formes démoniaques", in Satan, Coll. “Études Carmelitaines”, n. 6, Paris, Desclée de Brouwer, 1948, p. 510.

100. Campbell, The Moon-Lens, p. 49. Si tratta dell'unica occorrenza, nell'intero racconto, del pronome "lei", per quanto un'altra allusione alla femminilità di Shub-Niggurath emerga verso la fine di The MoonLens, quando la repellente metamorfosi di Leakey verrà descritta come "una rinascita nella vagina di Shub-Niggurath" (ibid., p. 52).

101. Il primo a menzionare un "Ariete dalle Mille Pecore" - pur non correlandolo a Shub-Niggurath - fu un brillante e intimo amico di Lovecraft, Clark Ashton Smith, all'inizio del racconto The Holiness of Azédarac (La santità di Azédarac, 1933); in seguito, diversi autori lovecraftiani lo adoperarono come epiteto alternativo di Shub-Niggurath (cfr. Daniel Harms, "Ram with a Thousand Ewes", in Id., Encyclopedia, p. 175).

102. Cfr. Chevalier, Gheerbrant, Dizionario, vol. II, p. 465. Sulla sintesi Luna-Terra-Madre, si veda Eliade, Trattato, p. 164. 
103. Eliade, Trattato, p. 155.

104. Murray, "On the Natures", p. 52.

105. Anthony Pearsall, "Shub-Niggurath”, in Id., The Lovecraft Lexicon: A Reader's Guide to Persons, Places and Things in the Tales of H. P. Lovecraft, Las Vegas (NV), New Falcon Publications, 2005, p. 457.

106. Campbell, The Moon-Lens, p. 48.

107. Ibid., p. 46, p. 49.

108. Come riconosciuto da Joshi, lo "scenario in cui il protagonista [...] è indotto ad alloggiare per la notte in un albergo è palesemente adattato da The Shadow over Innsmouth [di Lovecraft]" (Sunand T. Joshi, "Master and Pupil: August Derleth and Ramsey Campbell's First Book", in Crawford (ed.), Ramsey Campbell, p. 110).

109. Campbell, The Moon-Lens, p. 46.

110. Ibid.

111. Ibid.

112. Lévy, Lovecraft, p. 83.

113. Eliade, Trattato, p. 160.

114. Durand, Le strutture, p. 356.

115. Sul dio Proteo, si consulti Giulio Guidorizzi, Il mito greco, Milano, Mondadori, 2010, vol. I, p. 999 ss.

116. La fonte è un passo dell'invocazione alla dea lunare Ecate in Philosophumena (IV, 35) del Padre della Chiesa Ippolito "“Gorgo et Mormo et Luna et multiformis, / Venias propitia ad nostras libationes"; cfr. Jacques Paul Migne, Patrologia Graeca, vol 26, 3, cit. da Minerva, "La luna”, p. 40), conosciuta da Campbell probabilmente via Lovecraft, che la riproduce - anche lui, in traduzione - nella chiusura del racconto The Horror at Red Hook (L'orrore a Red Hook, 1927; cfr. Lovecraft, Tutti i romanzi e i racconti, p. 295).

117. Campbell, The Moon-Lens, p. 44.

118. Ibid., p. 48.

119. Cfr. ibid., p. 51.

120. Ibid.

121. Eliade, Trattato, p. 158-159.

122. Mircea Eliade, Mito e realtà, Torino, Borla, 1966, p. 109.

123. Campbell, The Moon-Lens, p. 153.

124. Ibid., p. 42.

125. Cfr. Lévy, Lovecraft, p. 76.

126. Robert M. Price, "Lovecraft's Concept of Blasphemy", in Id., H. P. Lovecraft, p. 6.

127. Durand, Le strutture, p. 387.

128. Campbell, The Moon-Lens, p. 53.

129. Eliade, Trattato, p. 168. 